\section{Robust Kalman Filter Design for Predictive Wind Shear Detection}

\author{
D. ALEXANDER STRATTON \\ ROBERT F. STENGEL, Fellow, IEEE \\ Princeton University
}

Severe low-altitude wind shear is a threat to aviation safety. Newly developed airborne sensors measure the radial component of wind along a line directly in front of an aircraft. In this paper, we use optimal estimation theory to define a detection algorithm to warn of hazardous wind shear from these sensors. To achieve robustness, a wind shear detection algorithm must distinguish threatening wind shear from less hazardous gustiness, despite variations in wind shear structure. Statistical analysis methods to refine wind shear detection algorithm robustness are presented. Computational methods predict the ability to warn of severe wind shear and avoid false warning. Comparative capability of the detection algorithm as a function of its design parameters is determined, identifying designs that provide robust detection of severe wind shear.
Manuscript received September 4, 1991; revised July 28, 1992.

IEEE Log No. T-AES/29/4/10983.

This research was supported by the NASA Langley Research Center under Grant NAG-1-834.

Authors' address: Dept. of Mechanical and Aerospace Engineering, Princeton University, Princeton, NJ 08540.

0018-9251/93/\$3.00 (C) 1993 IEEE

\section{INTRODUCTION}

An aircraft can deviate disastrously from its intended flight path if strong, varying wind (wind shear) is encountered near the ground. Severe low-altitude wind shear, such as produced by the microburst, has caused at least 24 aviation accidents in the last 25 years [1]. Efforts are underway to combat severe wind shear, including flight crew training [2], guidance strategies for wind shear encounter [3-5], and meteorological study of wind shear [6-8]. Ground-based sensor systems to measure airport-vicinity winds are being developed and installed at major airports $[9,10]$. While on-board reactive sensors are capable of detecting wind shear once it is encountered by an aircraft [11, 12], on-board forward-looking sensors are capable of sensing the winds directly in front of the aircraft, permitting a predictive detection of wind shear [13-15]. The refinement of techniques for predictive wind shear detection is the topic of this work.

Forward-looking sensors can provide advance warning of a wind shear threat, but wind measurements are influenced by moderate wind shear, turbulence, and measurement error. Optimal estimation theory $[16,17]$ provides a framework for minimizing the error of wind estimates given a hypothesis of the wind field structure. The wind shear detection algorithm presented here consists of multiple Kalman filters that exchange data to improve hazard estimates as the aircraft moves toward an area of wind shear. The algorithm predicts the level of an energy-based hazard metric from a series of wind measurements. Predictions can be used for hazard assessment by flight crews through a cockpit-based advisory system for wind shear avoidance $[18,19]$.

Prediction algorithm refinement must balance wind shear detection performance with the prevention of unnecessary warnings. Real wind shears will not possess the structure hypothesized in the development of the optimal estimators; therefore, algorithms must provide robust detection of severe wind shear. Recent research has focused on a statistical approach to the analysis of control system robustness [20]. Called stochastic robustness, this comprehensive approach to control system analysis uses statistical methods to compute probability-based robustness metrics. Statistical methods also have been used for sensitivity analyses of suboptimal estimators [21].

Statistical methods to analyze wind-shear-detectionalgorithm robustness are presented here. Three metrics, the probability of correct warning, the probability of false warning, and mean-square prediction error, are used to rate the robustness of the algorithm as a function of its design parameters. The probability of correct warning and mean-square prediction error are estimated with Monte Carlo methods, using an ensemble of representative wind shears generated 


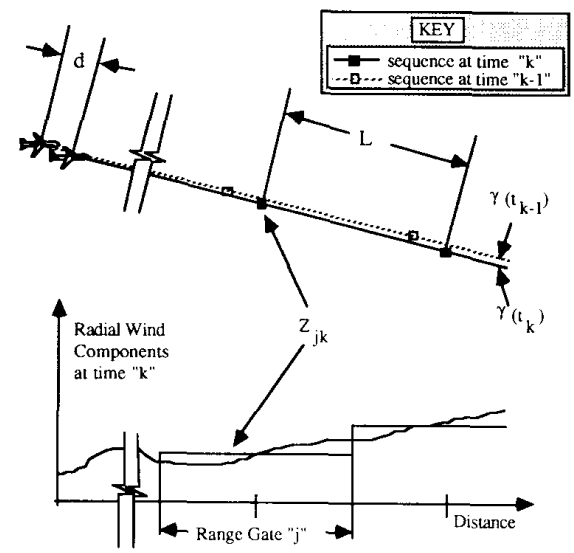

Fig. 1. Measurement process of forward-look sensor.

from empirical data [5] and a parametric microburst model [22]. The probability of false warning due to turbulence and measurement error also is estimated as a function of algorithm design parameters. The results quantify the tradeoff required between correct warning capability and false warning prevention, and they identify a design with robust detection characteristics.

\section{STOCHASTIC PREDICTION FOR WIND SHEAR DETECTION}

Airborne forward-look sensor technology currently under development is based on Doppler radar [13] and Doppler lidar [14] technology. One component of wind velocity can be measured by determining the shift in frequency of transmitted microwaves or light waves reflected from moisture or particulates. Operational devices could provide measurements of head winds or tail winds at a series of locations along the intended approach or takeoff path of the aircraft. For example, airborne Doppler radars can provide measurements spaced at $150 \mathrm{~m}$ intervals over a range of $6-10 \mathrm{~km}$, spanning 50-100 s of flight at approach speed [13]. At a given time, a sequence of measurements is obtained. A typical member of this sequence $z_{j k}$ represents the average value of the wind component along the radial $\gamma\left(t_{k}\right)$ in an interval of length $L$ at time $t_{k}$ (Fig. 1). As the aircraft travels down the flight path, measurements in successive sequences are offset by a distance $d$.

Prediction of aircraft hazard level is based on the air-referenced specific energy rate of the aircraft, which is defined by

$$
\frac{d E_{s}}{d t}(t)=\left(\frac{V_{a}}{g}\right) \frac{d V_{a}}{d t}+\frac{d h}{d t}
$$

$V_{a}$ is the airspeed, $h$ is aircraft altitude, and $g$ is the gravitational constant. Using longitudinal aircraft equations of motion and assuming small flight path angles, it can be shown [14] that

$$
\frac{d E_{s}}{d t}(t)=\frac{(T-D) V_{a}}{W}-\mathcal{F}(t) V_{a}
$$

where $T$ is thrust, $D$ is drag, and $W$ is aircraft weight. $\mathcal{F}(t)$ is the $F$-factor, defined as

$$
\mathcal{F}(t)=\left(\frac{1}{g}\right) \frac{d w_{x}}{d t}(t)-\frac{w_{h}(t)}{V_{a}}
$$

where $w_{x}(t)$ is the wind component in the inertial horizontal direction, and $w_{h}(t)$ is the vertical wind component. For small flight path angles, the radial wind components are approximately equal to the horizontal wind components. Wind shear effects enter (2) in three ways: by changing the airspeed, by altering the drag, and by determining $\mathcal{F}(t)$. For conditions typical of jet transport flight through severe wind shear, only the direct impact of $\mathcal{F}(t)$ is significant. Prediction of aircraft specific energy along the intended trajectory appears to involve the prediction of airspeed, but using a constant nominal value of airspeed in (3) introduces a small, conservative error.

Optimal estimators account for correlation in the measurement sequence using a stochastic model of the process being measured. The algorithm presented here assumes that $\mathcal{F}(t)$ is a process with a stationary mean, driven by a random input. With $\mathcal{F}(t)$ 's mean value stationary in space and time, the average $\mathcal{F}(t)$ across a particular range gate $j$ at time $k, \mathcal{F}_{j k}$, can be written as

$$
\mathcal{F}_{j k}=\left(\frac{L-d}{L}\right) \mathcal{F}_{j, k-1}+\left(\frac{d}{L}\right) \mathcal{F}_{j+1, k-1}+\eta_{j, k-1}
$$

where $\mathcal{F}_{j+1, k-1}$ is associated with the adjacent range gate further from the aircraft, and where $\eta$ is a normally distributed white noise sequence with zero mean and standard deviation $\sigma_{\eta j k}$. This standard deviation, which represents the prior uncertainty in horizontal wind shear and vertical winds, is a design parameter that influences the characteristic response of the algorithm. For the robustness study of this paper, fixed choices of $\sigma_{\eta}$ are considered to find a suitable range for this parameter. In real-time implementation, the algorithm can be used with fixed $\sigma_{\eta}$, or additional information sources (such as ground-based sensors) can be used to define a space and time-varying $\sigma_{\eta j k}$. The second alternative adapts the characteristic response of the algorithm for different circumstances.

An important limitation of Doppler sensors is their inability to measure winds perpendicular to the direction of the emitted radiation. As a consequence, the second component of $\mathcal{F}(t)$ in (3), due to vertical winds, is not measured by the device. In downburst wind shears, head-tail wind shear is produced by vertically descending winds that flow outward as 
they near the ground. These downdrafts pose a hazard to the aircraft that the Doppler sensors cannot measure directly. Techniques to quantify the relationship between horizontal and vertical winds for hazard estimation have been studied [23]. In the simple downburst model of [23], correlation between horizontal and vertical winds depends on the size of the downdraft, altitude, and distance from the downburst core. In a well-measured and studied microburst, four major downdraft regions were found [24]. For the algorithms presented here, vertical winds are assumed uncorrelated with the radial winds. If a consistent correlation is established between vertical winds and available measurements, vertical wind can be added to the state vector of the stochastic model.

The stochastic model is completed by defining the average radial wind component in range gate $j$ at time $k, w_{j k}$. Assuming piecewise-constant radial wind shear, $w_{j k}$ can be written as

$$
\begin{aligned}
w_{j k}= & w_{j, k-1}+\left[\frac{g(2 L-d) T_{s}}{2 L}\right] \mathcal{F}_{j, k-1} \\
& +\left(\frac{g d T_{s}}{2 L}\right) \mathcal{F}_{j+1, k-1}
\end{aligned}
$$

where $T_{s}$ is the time between measurement sequences. Because the wind shear is assumed to be stationary, the movement of the aircraft results in a dependency of the wind and wind shear close to the aircraft on the wind shear farther away. Defining the wind state at range gate $j$ by,

$$
\mathbf{x}_{j k}=\left[\begin{array}{ll}
w_{j k} & \mathcal{F}_{j k}
\end{array}\right]^{T}
$$

(4) and (6) may be rewritten in the vector-matrix form

$$
\mathbf{x}_{j k}=\Phi \mathbf{x}_{j, k-1}+\Phi_{C} \mathbf{x}_{j+1, k-1}+\Lambda \eta_{j, k-1} .
$$

Matrices of (7) are defined as

$$
\begin{gathered}
\Phi=\left[\begin{array}{cc}
1 & \left.\frac{g(2 L-d) T_{s}}{2 L}\right] \\
0 & \left(\frac{L-d}{L}\right)
\end{array}\right] \\
\Phi_{C}=\left[\begin{array}{ll}
0 & \left.\frac{g d T_{s}}{2 L}\right) \\
0 & \left(\frac{d}{L}\right)
\end{array}\right]
\end{gathered}
$$

and

$$
\Lambda=\left[\begin{array}{l}
0 \\
1
\end{array}\right] .
$$

Wind measurements obtained from a Doppler sensor give the speed of the wind relative to the speed of the aircraft. If the aircraft accelerates in a steady wind field, the wind measurements given by the Doppler device change with time in proportion to the change in airspeed. To avoid interpreting these accelerations as wind shear, a bias equal to the component of true aircraft airspeed parallel to the Doppler beam must be subtracted from the sensor measurements. With the assumption that measurement noise is superimposed on the radial wind components, the measurement at range gate $j$ at time $k, z_{j k}$, can be related to the corresponding scalar radial wind component by the relationship:

$$
z_{j k}=w_{j k}+n_{j k}+V_{a k} \cos \left(\alpha_{s k}\right) .
$$

This can be rewritten as

$$
\tilde{z}_{j k}=w_{j k}+n_{j k}
$$

where $V_{a k} \cos \left(\alpha_{s k}\right)$ is the component of true aircraft airspeed parallel to the Doppler beam at the time $k$, and $\tilde{z}_{j k}$ has this bias subtracted out:

$$
\bar{z}_{j k}=z_{j k}-V_{a k} \cos \left(\alpha_{s k}\right) .
$$

The measurement error $n_{j k}$ is assumed to be a zero mean, normally distributed white-noise sequence, with a known standard deviation $\sigma_{N j k}$. Equation (14) can be cast into the vector-matrix form,

$$
\tilde{z}_{j k}=H \mathbf{x}_{j k}+n_{j k}
$$

where

$$
H=\left[\begin{array}{l}
1 \\
0
\end{array}\right]
$$

Synthesis of an optimal estimation algorithm for the system of equations given by (7) and (14) proceeds by solving the discrete matrix Riccati equation for the optimal gain matrix associated with the wind state covariances [16]. If the total number of range gates is $J$, this solution appears to involve a $2 J \times 2 J$ nonlinear matrix equation, and the resulting estimator appears to be of order $2 J$. The solution can be partitioned into $J$ subsolutions, resulting in $J$ second-order estimators. The partitioned solution is more feasible for real-time implementation. The wind state estimate for range gate $j, \hat{\mathbf{x}}_{j k}(+)$, is computed by first extrapolating previous estimates to produce an extrapolated state, $\hat{\mathbf{x}}_{j k}(-)$, according to

$$
\hat{\mathbf{x}}_{j k}(-)=\Phi \hat{\mathbf{x}}_{j, k-1}(+)+\Phi_{C} \hat{\mathbf{x}}_{j+1, k-1}(+)
$$

and then performing a weighted sum of $\hat{\mathbf{x}}_{j k}(-)$ with the Doppler measurement using an optimal gain matrix:

$$
\hat{\mathbf{x}}_{j k}(+)=\hat{\mathbf{x}}_{j k}(-)+K_{j k}\left[\tilde{z}_{j k}-H \hat{\mathbf{x}}_{j k}(-)\right] .
$$

From [16], the optimal gain matrix for range gate $j$ at time $k$ is given by

$$
K_{j k}=P_{j k}(-)\left[\sigma_{N j k}^{2}+H P_{j k}(-)^{-1} H^{T}\right]^{-1}
$$

where $P_{j k}(-)$, the extrapolated covariance matrix of range gate $j$ at time $k$, is defined as

$$
P_{j k}(-)=E\left\{\mathbf{x}_{j k}(-) \mathbf{x}_{j k}(-)^{T}\right\}
$$


where $E\{\cdot\}$ denotes the expected or mean value. This covariance can be extrapolated from covariance matrices, $P_{j, k-1}(+)$ and $P_{j+1, k-1}(+)$, by the extrapolation equation

$$
\begin{aligned}
P_{j k}(-)= & \Phi P_{j, k-1}(+) \Phi^{T}+\Phi_{C} P_{j+1, k-1}(+) \Phi_{C}^{T} \\
& +\Lambda \sigma_{\eta j k}^{2} \Lambda^{T} .
\end{aligned}
$$

The covariance matrix is updated to produce $P_{j k}(+)$ according to the standard form:

$$
P_{j k}(+)=\left[P_{j k}(-)+H^{T} \sigma_{N j k}^{-2} H\right]^{-1} .
$$

The algorithm given by (16)-(21) can be implemented by a set of $J$ processors running in parallel. Each processor updates the state estimate and covariance matrix of a given range gate, obtaining a previous state estimate and updated covariance from the processor of the neighboring range gate farther from the aircraft. As the aircraft approaches an area of wind shear, estimates of the hazard level are passed from processor to processor, improving the accuracy of the estimate. Wind and hazard estimates from the algorithm can be combined with reactive sensor measurements in similar manner, improving the timeliness of reactive wind shear alerts. If multiple sensors are available, the algorithm takes the same form, except that $\tilde{z}_{j k}$ becomes a vector, while $\sigma_{N j k}^{2}$ becomes a matrix.

For the outermost range gate, $j=J$, the algorithm takes a different form. Lacking another prediction of the wind state in the region beyond the range of the sensor, the state and covariance are extrapolated according to

$$
\begin{aligned}
& \hat{\mathbf{x}}_{J k}(-)=\left(\Phi+\Phi_{C}\right) \hat{\mathbf{x}}_{J, k-1}(+) \\
& P_{J k}(-)=\left(\Phi+\Phi_{C}\right) P_{J, k-1}(+)\left(\Phi+\Phi_{C}\right)^{T}+\Lambda \sigma_{\eta J k}^{2} \Lambda^{T}
\end{aligned}
$$

If predictions of wind state beyond the range of the sensor are available from other sensors, (16)-(21) can be used.

\section{SIMULATION OF STOCHASTIC PREDICTION}

A batch simulation of a downburst wind shear demonstrates the response of the algorithm and demonstrates the sensitivity to the design parameter $\sigma_{\eta}$. For the simulation, two different predictions are made, each with different constant choices of the design parameter $\sigma_{\eta}$. The wind shear is modeled by the Oseguera-Bowles stagnation-point-flow downburst model [22]. Turbulence is superimposed on the wind shear using the horizontal Dryden spectrum [25]. Gaussian, zero-mean white noise is superimposed on the wind field models to simulate Doppler sensor measurement error. The wind shear simulation is
TABLE I

Simulation Parameters for Algorithm Evaluation

Aircraft Initial Conditions

Airspeed, $V_{a}$

Altitude, $h$

$160 \mathrm{kt}$

$2000 \mathrm{ft}$

Inertial flight path angle, $\gamma_{i}$

Distance to microburst core

$-3 \mathrm{deg}$

$20,100 \mathrm{ft}$

\section{Doppler Sensor}

Range gate separation, $L$

Distance between sequences, $d$

Time between sequences, $T_{s}$

Noise standard deviation, $\sigma_{N}$

Range

$0.1 \mathrm{~s}$

$0.1 \mathrm{~s}$
$1 \mathrm{ft} / \mathrm{s}$

$20,000 \mathrm{ft}$

Turbulence

Rms turbulence intensity, $\sigma_{u}$

Turbulence Scale length, $L_{u}$

$2.7 \mathrm{ft} / \mathrm{s}$

$1000 \mathrm{ft}$

Downdraft radius

Microburst

Maximum horizontal winds

Height of Boundary Layer

$2070 \mathrm{ft}$

$58.4 \mathrm{ft} / \mathrm{s}$

$131 \mathrm{ft}$

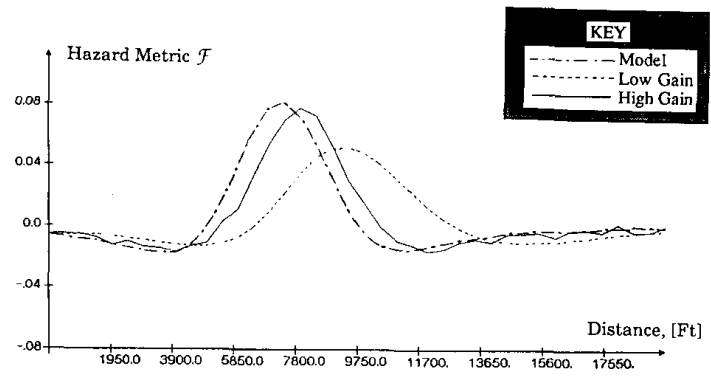

Fig. 2. Simulation of stochastic prediction algorithm.

initiated with the microburst approximately $10,000 \mathrm{ft}$ in front of the aircraft. Other parameters of the simulation are presented in Table I.

Fig. 2 compares the predicted hazard metric $\hat{\mathcal{F}}$ along the flight path with the $\mathcal{F}(t)$ component of the model due to the head-tail wind shear alone, ten seconds after the start of the simulation. The predictions agree well with the model's head-tail wind component of $\mathcal{F}(t)$, but the peak magnitude of the prediction is attenuated due to the finite bandwidth of the prediction algorithm. In addition, the distance between the aircraft and the wind shear is over-predicted due to filter lag. For the algorithm with lower value of $\sigma_{\eta}$, which has lower gains, these effects are more pronounced. However, the algorithm with lower $\sigma_{\eta}$ attenuates turbulence and measurement error effects more effectively. The choice of $\sigma_{\eta}$ requires a compromise between turbulence rejection and attenuation of severe wind shear. Variation in the size and shape of severe wind shear complicate this choice.

If it is desirable to warn whenever $\mathcal{F}(t)$ exceeds a given level $T$, a warning could be issued whenever $\hat{\mathcal{F}}$ exceeds a design threshold level, $T_{d}$. If this design threshold is set equal to $T$, the effect of unmeasured 
vertical winds and attenuation could result in the system not warning when approaching a wind shear that exceeds $T$-a missed detection. However, lowering the design threshold makes a warning due to turbulence or measurement error when severe wind shear is not present (a false warning) more likely. The design threshold level $T_{d}$ also influences the likelihood of false warning and missed detection. A means to quantify the quality of detection algorithms and to identify robust designs is the topic of the remainder of this work.

\section{STOCHASTIC DETECTION ROBUSTNESS METRICS}

The prediction algorithm stochastic model is based on constant wind shear, with variation in the level, or structure, of the wind shear modeled by white noise. In actual wind shear, a variety of possible structures exist. The ability to distinguish hazardous wind shear from nonhazardous disturbances, despite the variation in wind shear structure, is called detection robustness. The optimization of detection robustness must take into account both missed detection and false-warning prevention. A robust algorithm responds to areas of sustained, significant wind shear, and not to brief or mild disturbances that do not affect the flight path. To rate the robustness of candidate designs, quantitative measures of detection robustness are needed.

The use of probability-based metrics for control system robustness is presented in [20]. For example, the probability of instability was introduced as a measure of linear multivariable control system robustness. For detection robustness, three metrics are used: the probability of correct warning, the probability of false warning, and mean-square prediction error in severe wind shear. The first two metrics are measures of the system's reliability while the third is a measure of the system's accuracy. The robustness metrics defined are computed as functions of the stochastic prediction design parameters and of the design threshold level above which a warning would be generated.

To illustrate the importance of the first two metrics, let $H$ represent a hypothesis of severe wind shear on the intended flight path of an aircraft. Let $E$ represent a warning generated by a detection algorithm, perhaps based on the exceedence of a threshold level of a hazard prediction. Given a prior probability of encounter with severe wind shear, $\operatorname{Pr}\{H\}$, the probability of severe wind shear can be updated or conditioned on the warning using Bayes's rule,

$$
\operatorname{Pr}\{H \mid E\}=\frac{\operatorname{Pr}\{E \mid H\}}{\operatorname{Pr}\{E\}} \operatorname{Pr}\{H\}
$$

where $\operatorname{Pr}\{H \mid E\}$ is called the posterior probability of $H$. From the axioms of probability, the prior probability of receiving the alarm, $\operatorname{Pr}\{E\}$, can be expressed as,

$$
\operatorname{Pr}\{E\}=\operatorname{Pr}\{E \mid H\} \operatorname{Pr}\{H\}+\operatorname{Pr}\{E \mid \neg H\}[1-\operatorname{Pr}\{H\}]
$$

where " $\neg H$ " denotes the logical negation of $H$, i.c., no severe wind shear on flight path. $\operatorname{Pr}\{E \mid H\}$ is the probability of correct warning or $\mathbb{P}_{\mathrm{CW}}$, while $\operatorname{Pr}\{E \mid \neg H\}$ is the probability of false warning or $P_{F W}$. Note that the probability of missed detection, $\operatorname{Pr}\{\neg E \mid H\}$, is related to $\mathbb{P}_{\mathrm{CW}}$ by

$$
\operatorname{Pr}\{\neg E \mid H\} \equiv \mathbb{P}_{\mathrm{MD}}=1-\mathbb{P}_{\mathrm{CW}} .
$$

The third metric, mean-square prediction error, measures the accuracy of the prediction algorithm in a severe wind shear environment. For a single range gate $j$ of the filter for $K$ steps of a discrete simulation, mean-square prediction error, $\mathbb{E}_{j}$, is defined as

$$
\mathbb{E}_{j}=\frac{1}{K} E\left\{\sum_{k=1}^{K}\left[\hat{\mathcal{F}}_{j k}(+)-\mathcal{F}\left(t_{k}\right)_{a v e_{j k}}\right] \mid H\right\}
$$

where $\mathcal{F}\left(t_{k}\right)_{\text {ave } e_{j k}}$ denotes the value of the actual $\mathcal{F}(t)$ at the time step $k$, averaged over the location of range gate $j$ at time step $k$. Here, $E\{\cdot \mid H\}$ denotes the expected or mean value given severe wind shear. The evaluation of probability of correct warning and mean-square prediction error are evaluated in a Monte Carlo simulation of downburst wind shear.

\section{MONTE CARLO SIMULATION OF DOWNBURST ENCOUNTERS}

Monte Carlo simulation is a means of analyzing systems described by random variables. The simulation consists of a large number of tests, with values for the random variables selected from corresponding probability distributions. Each test in a Monte Carlo simulation of wind shear detection is an encounter with downburst wind shear. Uncertainty in wind shear structure is represented by an analytical model whose parameters are random variables. The Oseguera-Bowles downburst model, which was developed to match vertical and horizontal wind profiles of empirical data, is used to simulate the wind shear structure. The equations of this model are functions of three parameters: the maximum horizontal winds $u_{m}$, outflow radius $R_{0}$, and altitude of maximum outflow $z_{m}$. Probability distributions of maximum horizontal winds and outflow radius $R_{0}$ are taken from a survey based on nationwide meteorological studies of downbursts [8] and reproduced here as Fig. 3. The probability distribution of $z_{m}$ is assumed to be uniform between 60 and $160 \mathrm{~m}$. 


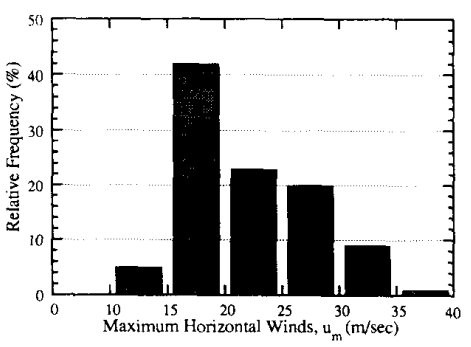

(a)

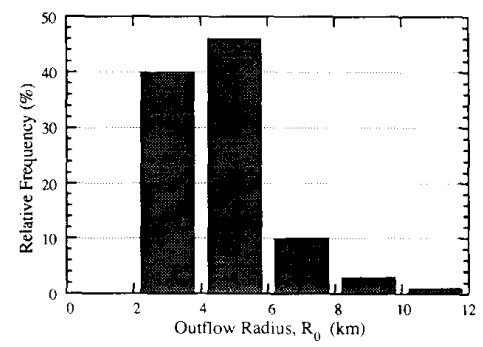

(b)

Fig. 3. Relative frequency of outflow radius and maximum horizontal winds from nationwide survey of downbursts [8]

An ensemble of downburst wind shears is generated by picking random values of $u_{m}, R_{0}$, and $z_{m}$ from these distributions. Turbulence is superimposed on the model using the horizontal Dryden spectrum, with a constant length scale $l_{u}$ of $1000 \mathrm{ft}$ and intensity $\sigma_{u}$ of $2.7 \mathrm{ft} / \mathrm{s}$. While this ensemble does not span the entire range of wind shear structures, the ensemble is adequate for comparing the stochastic robustness of candidate designs. Constant choices of $\sigma_{\eta}$ are used to define several different detection algorithms. Sensor measurement error characteristics are the same as in the simulation above.

To test the detection algorithm, each trajectory is initiated at a distance from the downburst well exceeding the range of the sensor. Altitudes of the level-flight trajectories are picked uniformly between the boundary layer altitude $z_{m} / 2$, and $1200 \mathrm{ft}$. The trajectories are offset from the axis of symmetry of the downburst; the offset distance is picked uniformly ranging from 0 to $R_{0}$. Each trajectory is run until the aircraft reaches the edge of the downdraft, with the aircraft moving at a constant inertial speed of $160 \mathrm{kt}$.

Each measurement sequence is simulated and processed by the detection algorithms every $0.1 \mathrm{~s}$, or equivalently, every $27 \mathrm{ft}$. The hazard predictions are averaged over five range gates, and a warning criterion based on the hazard predictions is checked for each of several design threshold levels $T_{d}$ : if any of the averages exceeds the threshold, a warning is declared.
The warning criterion can be expressed as

$$
\frac{1}{5} \sum_{m=j}^{j+4} \hat{\mathcal{F}}_{m k}(+) \equiv \hat{\mathcal{F}}_{a v e_{j k}}>T_{d} .
$$

A downburst wind shear encounter may or may not involve a hazardous level of wind shear. For each wind shear, a truth criterion is checked to see whether or not that wind shear is hazardous. The NASA Langley criterion for hazardous wind shear is used: if the average value of the actual $\mathcal{F}(t)$ over $1 \mathrm{~km}$ exceeds a threshold for 0.1 , the shear is hazardous [15]. Of the 2200 simulated downburst encounters, 978 (44\%) did not involve a hazardous level of wind shear, according to the NASA definition.

Detection robustness metrics can be estimated from the Monte Carlo simulation. For a large number of downburst wind shear encounters, the probability of correct warning may be approximated by,

$$
\mathbb{P}_{\mathrm{CW}} \approx \frac{N_{\mathrm{CW}}}{N_{\mathrm{SW}}}
$$

where $N_{\mathrm{CW}}$ is the number of correct warnings of severe wind shear, and $N_{\mathrm{SW}}$ is the number of severe wind shears in the ensemble. Mean-square prediction error is computed for the warning criterion, $\hat{\mathcal{F}}_{a v e_{j k}}$, by

$$
\mathbb{E}_{j} \approx \frac{1}{N_{\mathrm{SW}}} \sum_{n=1}^{N_{\mathrm{SW}}}\left\{\frac{1}{K} \sum_{k=1}^{K}\left[\hat{\mathcal{F}}_{\text {ave }_{j k}}-\mathcal{F}\left(t_{k}\right)_{a v e_{j k}}\right]\right\} .
$$

The estimated probability of correct warning, $P_{C W}$, is plotted in Fig. 4 as a function of the design threshold level, for two different choices of $\sigma_{\eta}$. The probability of correct warning increases by lowering the design threshold and raising $\sigma_{\eta}$. At a design threshold equal to 0.1 , the probability of correct warning is less than $50 \%$, due to the unmeasured vertical winds. Only $48 \%$ of the severe wind shear encounters simulated would meet the NASA Langley criterion based on the head-tail wind component of $\mathcal{F}(t)$ alone. Thus, an error-free predictor of the head-tail wind shear would have a probability of correct warning of $48 \%$ at a design threshold of 0.1 . For a higher probability of correct warning, Doppler-sensor-based warnings must be based on a lower threshold, or equivalently, $\mathcal{F}$ predictions must be scaled upward. $\mathcal{F}$ predictions from the algorithm of this paper exceed 0.1 for at least $99 \%$ of the wind shear encounters in which the head-tail wind component of $\mathcal{F}(t)$ exceeds 0.1 .

Mean-square prediction error provides the basis for selecting the design parameter, $\sigma_{\eta}$. For the parameters of Table I, the estimated mean-square prediction error, $\mathbb{E}_{j}$, has a minimum where $\sigma_{\eta} \approx 0.0025$ (Fig. 5). For values below the minimum, attenuation effects cause an increase in error, while above the minimum, the error is increased by amplification of noise and turbulence. 


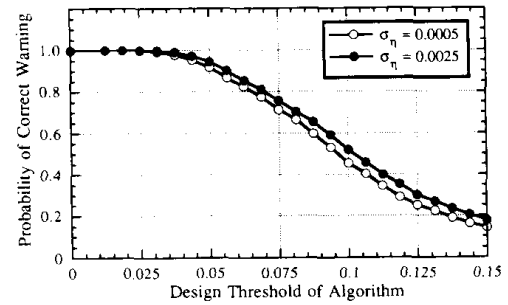

Fig. 4. Probability of exceeding design threshold in severe downburst wind shear for two different values of $\sigma_{\eta}$.

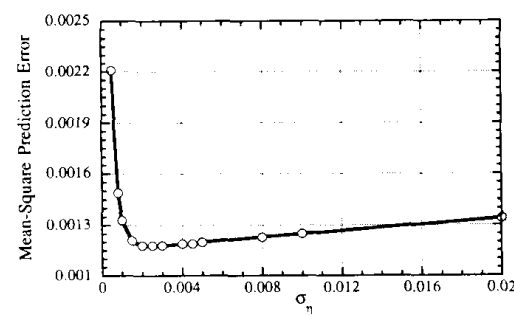

Fig. 5. Selection of design parameter $\sigma_{\eta}$ for minimum mean-square $\mathcal{F}(t)$ prediction error in severe downburst wind shear.

\section{ANALYSIS OF FALSE WARNINGS}

Estimates of the probability of false warning, $\mathbb{P}_{\mathrm{FW}}$, are computed as functions of the design parameters. The computation is based on finding the steady-state rate of false warnings, which occurs whenever the design threshold is crossed due to turbulence or measurement noise. For a statistically stationary, zero-mean Gaussian process, it can be shown [26] that the number of times the output $y(t)$ crosses a threshold, $T_{d}$, per unit time is given by

$$
N\left(T_{d}\right)=\frac{\sigma_{\dot{y}}}{2 \pi \sigma_{y}} e^{-\left(T_{d}^{2} / 2 \sigma_{y}^{2}\right)} .
$$

Correspondingly, if turbulence and noise inputs are assumed stationary, zero-mean Gaussian processes, the steady-state rate at which a prediction $\hat{\mathcal{F}}_{a v e_{j k}}$ crosses a threshold $T_{d}, N\left(T_{d}\right)$, is given by,

$$
N\left(T_{d}\right)=\frac{\sigma_{\mathcal{F}}}{2 \pi \sigma_{\mathcal{F}}} e^{-\left(T_{d}^{2} / 2 \sigma_{\mathcal{F}}^{2}\right)}
$$

where $\sigma_{\mathcal{F}}^{2}$, the variance in the hazard prediction, is defined by

$$
\sigma_{\mathcal{F}}^{2}=E\left\{\hat{\mathcal{F}}_{a v e_{j k}}^{2}\right\} .
$$

With a sample time of $T_{s}$, the variance of change in the hazard prediction, $\sigma_{\dot{\mathcal{F}}}^{2}$, is given by

$$
\begin{aligned}
\sigma_{\dot{\mathcal{F}}}^{2} & =E\left\{\left[\frac{d}{d t}\left(\hat{\mathcal{F}}_{a v e}\right)\right]^{2}\right\} \\
& =\frac{E\left\{\left[\hat{\mathcal{F}}_{a v e_{j k}}-\hat{\mathcal{F}}_{a v e_{j k-1}}\right]^{2}\right\}}{T_{S}}
\end{aligned}
$$

and $\sigma_{\mathcal{F}}$ and $\sigma_{\dot{\mathcal{F}}}$ are evaluated for stochastic equilibrium as $k \rightarrow \infty$ using a steady-state Riccati equation. Since the gains are fixed at steady-state values, the Joseph form of this Riccati equation is most convenient [16]:

$$
\begin{aligned}
P_{j}(+)= & \left(I-K_{j} H\right)\left[\Phi P_{j}(+) \Phi^{T}+\Phi_{C} P_{j+1}(+) \Phi_{C}^{T}\right] \\
& \times\left(I-K_{j} H\right)^{T}+K_{j} \sigma^{2} K_{j}^{T}
\end{aligned}
$$

where $\sigma^{2}$ is the sum of turbulence and measurement error variances:

$$
\sigma^{2}=\sigma_{t}^{2}+\sigma_{N}^{2} .
$$

Equation (36) defines a $2 J \times 2 J$ system of linear, algebraic equations, and it can be solved for $\sigma=1$, to define a constant of proportionality between $\sigma$ and prediction algorithm output standard deviations:

$$
\begin{aligned}
\sigma_{\mathcal{F}} & =C_{1} \sigma \\
\sigma_{\dot{\mathcal{F}}} & =C_{2} \sigma .
\end{aligned}
$$

Combining these results with (34),

$$
N\left(T_{d}\right)=\frac{C_{2}}{2 \pi C_{1}} e^{-\left(T_{d}^{2} / 2 C_{1}^{2} \sigma^{2}\right)} .
$$

Equation (39) is valid for a given $\sigma_{t}$; however, the intensity of turbulence is known to vary. Thus, the expected value of $N\left(T_{d}\right)$ is taken over the variation in $\sigma_{t}$ :

$$
E\left\{N\left(T_{d}\right)\right\}=\int_{0}^{\infty} \frac{C_{2}}{2 \pi C_{1}} e^{-\left(T_{d}^{2} / 2 C_{1}^{2} \sigma^{2}\right)} \operatorname{pr}\left(\sigma_{t}\right) d \sigma_{t} .
$$

From [25], the probability density of turbulence intensity is given by a Rayleigh density,

$$
\operatorname{pr}\left(\sigma_{t}\right)=\frac{\sigma_{t}}{c} e^{-\left(\sigma_{t}^{2} / 2 c^{2}\right)} \quad \sigma_{t}>0
$$

where $c=2.3 \mathrm{ft} / \mathrm{s}$. Equation (40) can be integrated numerically for the value of the prediction algorithm design parameters. Finally, the probability of false warning is computed from $E\left\{N\left(T_{d}\right)\right\}$ using the Poisson distribution,

$$
\mathbb{P}_{\mathrm{FW}}=1-e^{-t_{T} E\left\{N\left(T_{d}\right)\right\}}
$$

where $t_{T}$ is the average time of a terminal operation, taken as $60 \mathrm{~s}$.

\section{DESIGN FOR DETECTION ROBUSTNESS}

$P_{\text {FW }}$ and the probability of missed detection are shown in Fig. 6 as functions of threshold level for $\sigma_{\eta}$ equal to 0.0025 . Selection of a design threshold requires a compromise between these two factors. Two methods for choosing a design threshold based on the robustness analysis can be specified here. First, if a maximum operator tolerance for false alarms can be 


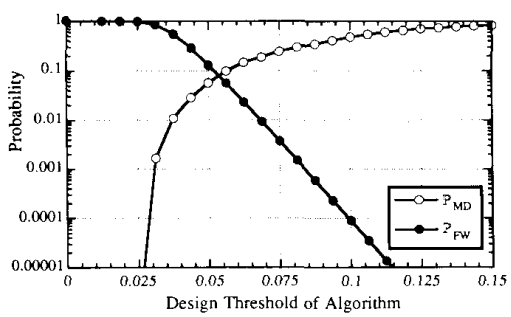

Fig. 6. Missed detection and false alarm design tradeoff.

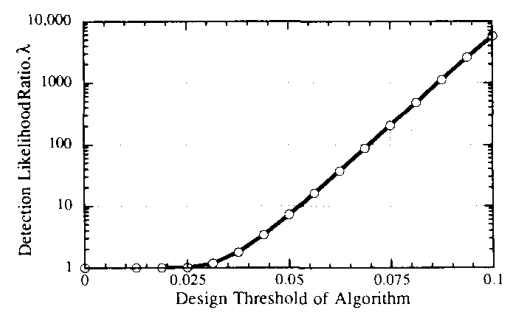

Fig. 7. Detection likelihood ratio versus design threshold.

specified, the threshold can be set on the basis of $P_{F W}$ alone. If the probability of severe wind shear encounter must be kept below a given standard, the threshold can be selected on the basis of detection likelihood ratio $\lambda$, which is defined by

$$
\lambda=\frac{\mathbb{P}_{\mathrm{CW}}}{\mathbb{P}_{\mathrm{FW}}} .
$$

From Bayes's rule, (24), it can be shown that

$$
\lambda=\frac{\operatorname{Pr}\{H \mid E\}}{[1-\operatorname{Pr}\{H \mid E\}]} \frac{[1-\operatorname{Pr}\{H\}]}{\operatorname{Pr}\{H\}} .
$$

If a maximum tolerable $\operatorname{Pr}\{H \mid E\}$ is specified and the prior probability of wind shear encounter $\operatorname{Pr}\{H\}$ is known, the minimum level of $\lambda$ is found from (44). The threshold is picked from a plot of $\lambda$ versus design threshold (Fig. 7).

If multiple evidence is available, (44) and Fig. 7 can be used to adapt a varying design threshold on the basis of that evidence, by interpreting the prior probability $\operatorname{Pr}\{H\}$ as the conditional probability of wind shear encounter given all other evidence except the forward-look sensor measurements. Prior evidence that supported the presence of wind shear would lower the threshold for warning. A Bayesian method to compute $\operatorname{Pr}\{H\}$ has been described previously in [18]. When the prediction algorithm is integrated into a probability-based reasoning system, a set of multiple thresholds can be employed; exceedence of these thresholds would indicate multiple levels of support for the probability of severe wind shear.

\section{CONCLUSIONS}

Optimal estimation provides a framework for hazard prediction from airborne Doppler sensors.
Given a hypothesis of the local wind field, a bank of parallel Kalman filters estimates hazard level, optimally weighing current and prior measurements and compensating for the movement of the sensor platform. Multiple sensors and combined predictive and reactive wind shear detection are natural extensions to the algorithm. A statistical approach to refining robustness takes advantage of available empirical evidence and an analytical model.

Many extensions to the design and analysis methods of this paper could yield improved detection performance. For example, improved statistical models could be developed that accurately model the dynamic development and three-dimensional wind structure of a microburst. These stochastic analysis methods could also be applied to the development of robust wind shear recovery guidance strategies. Near-optimal control policies could be developed that minimize stochastic cost function penalties over variations in wind shear intensity and structure.

The availability of an on-board sensor should not result in missed opportunities to avoid the hazard altogether. Furthermore, limitations of a single sensor can be partially overcome by utilizing other indicators to build confidence that an encounter with wind shear is imminent. If a Doppler sensor is used as a sole source of wind shear information, keeping the probability of wind shear encounter at an acceptable level dictates a fixed threshold, fixing the false warning rate. If multiple sources of information are effectively integrated, a variable threshold or multiple thresholds can be employed to maintain the same or lower probability of wind shear encounter with a lower false warning rate.

\section{REFERENCES}

[1] Townsend, J. (1993)

Low-Atitude Wind Shear and Its Hazard to Aviation. Washington, DC: National Academy Press, 1983.

[2] FAA (1987)

Windshear Training Aid.

U.S. Department of Transportation, Federal Aviation Administration, Associate Administrator for Development and Logistics, Washington, DC, Feb. 1987.

[3] Psiaki, M. L., and Stengel, R. F. (1986) Optimal flight paths through microburst wind profiles. Journal of Aircraft, 23, 8 (Aug. 1986), 629-635.

[4] Hinton, D. A. (1989)

Piloted simulation of recovery guidance for microburst windshear encounters.

NASA technical paper 2886, DOT/FAA/DS-89/06, Washington, DC, Mar. 1989.

[5] Miele, A., Wang, T., and Melvin, W. (1986) Optimization and Gamma/Theta guidance of flight trajectories in a windshear. In Proceedings of the 15th Congress of the International Council of the Aeronautical Sciences, London, England, Sept. 1986, 878-899; ICAS-86-5.6.4. 
[6] Fujita, T. T. (1985)

The downburst: Microburst and macroburst.

University of Chicago, Chicago, IL, Satellite and Mesometeorology Research Project, 1985.

[7] McCarthy, J., Roberts, R., and Schreiber, W. (1983) JAWS data collection, analysis highlights, and microburst statistics.

In Proceedings of the 21st Conference on Radar Meteorology, American Meteorological Society, 1983, 596-601.

[8] Wolfson, M., et. al. (1990)

Characteristics of thunderstorm-generated low-altitude wind shear: A survey based on nationwide Doppler weather radar testbed measurements. In Proceedings of the 29th Conference on Decision and Control, Vol. 2, Honolulu, HI, 1990, 682-688.

[9] Turnbull, D., McCarthy, J., Evans, J., and Zrnic', D. (1989) The FAA terminal Doppler weather radar (TDWR) program.

In Praceedings of the 3rd International Conference on the Aviation Weather System, Anaheim, CA, Feb. 1989, $414-419$.

[10] Barab, J. D., Page, R. D., Rosenburg, B. L., Zurinskas, T. E., and Smythe, G. R. (1988)

Evaluation of enhancements to the low level windshear alert system (LLWAS) at Stapleton International Airport. DOT/FAA/PS-88/14, Springfield, VA, July 1987-Mar. 1988.

[11] Saint, S. (1983)

The missing element in wind shear protection.

Business Aircraft Meeting and Exposition, Wichita, KA, Apr. 1983; SAE 830715

[12] Zweifel, T. (1988)

Sensor consideration in the design of a windshear detection and guidance system.

Aerospace Technology Conference and Exposition,

Anaheim, CA, Oct. 1988; SAE 881417.

[13] Bracalente, E. M., and Jones, W. R. (1990)

Airborne Doppler radar detection of low altitude windshear.

Journal of Aircraft, 27, 2 (Feb. 1990), 151-157.

[14] Targ, R., and Bowles, R. L. (1988)

Airborne LIDAR for avoidance of windshear hazards.

In Proceedings of the Second Combined Manufacturer's and

Technology Airborne Windshear Review Meeting, Vol. 1, Williamsburg, VA, Oct. 1988, 369-377.

[15] Bowles, R. L. (1990)

Windshear detection and avoidance: Airborne systems survey.

In Proceedings of the 29th Conference on Decision and Control, Vol. 2, Honolulu, HI, Dec. 1990, 708-736.
[16] Stengel, R. F. (1986)

Stochastic Optimal Control.

New York: Wiley, 1986.

[17] Anderson, B. D. O., and Moore, J. B. (1979) Optimal Filtering.

Englewood Cliffs, NJ: Prentice-Hall, 1979.

[18] Stratton, D. A., and Stengel, R. F. (1990)

Probabilistic reasoning for intelligent wind shear avoidance.

In Proceedings of the 1990 ALAA Guidance, Navigation, and Control Conference, Vol. 2, Portland, OR, Aug. 1990 1099-1107.

[19] Stengel, R. F, and Stratton, D. A. (1989)

An expert system for wind shear avoidance.

Engineering Applications of Artificial Intelligence, 2, 3

(Sept. 1989), 190-197.

[20] Ray, L. (1991)

Stochastic robustness of linear multivariable control systems: Towards comprehensive robustness analysis. Ph.D. dissertation, report 1902-T, Princeton University, Princeton, NJ, Jan. 1991.

[21] Gelb, A. (1974) Applied Optimal Estimation.

The Analytic Sciences Corporation, Washington, DC 1974.

[22] Oseguera, R., and Bowles, R. L. (1988)

A simple analytic, 3-dimensional downburst model based on boundary layer stagnation flow.

TM 100632, NASA, Washington, DC, July 1988.

[23] Byrd, G. P., Proctor, F. H., and Bowles, R. L. (1990) Evaluation of a technique to quantify microburst windshear hazard potential to aircraft. In Proceedings of the 29th Conference on Decision and Control, 2, Honolulu, HI, Dec. 1990, 689-694.

[24] Proctor, F. (1990)

Model comparison of July 7, 1990 microburst. In Proceedings of the Third Combined Manufacturer's and Technology Airborne Windshear Review Meeting, Vol. 1, Hampton, VA, Oct. 1990, 81-103.

[25] (1980)

Flying Qualities of Piloted Airplanes. Military Specification 8785, Nov. 1980.

[26] Rice, S. O. (1954)

Mathematical analysis of random noise.

In Selected Papers on Noise and Stochastic Processes. New York: Dover, 1954 


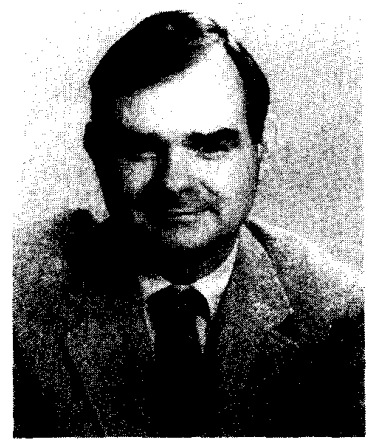

Alex Stratton received the B.S. in aeronautical engineering from Rensselaer Polytechnic Institute, Troy, NY, in 1985, and the Ph.D. in mechanical and aerospace engineering from Princeton University, Princeton, NJ, in 1992.

From 1985 to 1987 he was employed in Flight Control Systems Development at Grumman Aircraft Systems Division. He is currently employed at Parker-Hannifin Corp., Gull Electronics Division in Smithtown, NY. His interests include airborne navigation, flight inspection, estimation and control theory, and artificial intelligence.

Robert Stengel (M'77-SM'83-F'93) received degrees from Massachusetts Institute of Technology, Cambridge, MA (aeronautics and astronautics, S.B., 1960) and Princeton University, Princeton, NJ, (aerospace and mechanical sciences, M.S.E., M.A., Ph.D., 1965, 1966, 1968).

$\mathrm{He}$ is currently Professor of Mechanical and Aerospace Engineering at Princeton University, Princeton, NJ, where he directs the Topical Program on Robotics and Intelligent Systems and the Laboratory for Control and Automation. Prior to his 1977 Princeton appointment, he was with The Analytic Sciences Corporation, Charles Stark Draper Laboratory, U.S. Air Force, and National Aeronautics and Space Administration. A principal designer of the Project Apollo Lunar Module manual attitude control logic, he also contributed to the design of the Space Shuttle guidance and control system. His current research focuses on system dynamics, control, and machine intelligence. He teaches courses on control and estimation, aircraft dynamics, space flight engineering, and acrospace guidance.

Dr. Stengel is an Associate Fellow of the AIAA, and a Member of the SAE Aerospace Control and Guidance Systems Committee. Professional positions include Associate Editor at Large of the IEEE Transactions on Automatic Control, North American editor of the Cambridge University Press Aerospace Series, and Member of the Program Council for the New Jersey Space Grant Consortium. He was Vice Chairman of the Congressional Aeronautical Advisory Committee and Chairman of the AACC Awards Committee, and he has served on numerous governmental advisory committees. He wrote the book, Stochastic Optimal Control: Theory and Application, (Wiley, 1986) and is writing a book on aircraft dynamics and control. 